02

\title{
Фурье-спектроскопия как метод изучения фотоэлектрических свойств органических систем
}

\author{
() С.П. Палто, А.В. Алпатова, А.Р. Гейвандов, Л.М. Блинов, В.В. Лазарев, С.Г. Юдин \\ Институт кристаллографиии им. А.В. Шубникова, ФНИЦ „Кристаллографрия и фотоника“ РАН, \\ 119333 Москва, Россия \\ e-mail: serguei.palto@gmail.com
}

Поступила в редакцию 15.09.2017 г.

Предложен новый метод изучения фотоэлектрических свойств слоистых тонкопленочных структур, основанный на широкополосной Фурье-спектроскопии, где модуляция оптической задержки осуществляется по гармоническому закону. В отличие от традиционных подходов изучения фотоэлектрических свойств с применением дисперсионных спектральных приборов предлагаемый метод позволяет не только одновременно охватывать ультрафиолетовую, видимую и инфракрасную области спектра, обеспечивая при этом широкий динамический диапазон и спектральное разрешение, но и легко варьировать низкочастотную модуляцию светового воздействия. Возможности метода продемонстрированы на примере исследования поликристаллической органической гетероструктуры; измерены ее спектральная чувствительность, быстродействие и удельная обнаружительная способность. Предложены модель и эквивалентная электрическая схема для объяснения результатов измерений.

DOI: $10.21883 /$ OS.2018.02.45526.209-17

\section{Введение}

В последнее время органические микро- и наноструктуры все больше используются в (микро-) наноэлектронике и фотонике, конкурируя с системами на основе неорганических полупроводников. Наглядным примером являются жидкие кристаллы [1] и светоизлучающие органические гетероструктуры [2], а также информационные дисплеи и телевизионные панели на их основе. Наряду с такими системами особое внимание уделяется и структурам для преобразования световой энергии в электрическую [3], которые, в свою очередь, могут использоваться для различных целей, таких как преобразование солнечной энергии в электрическую [4]; детектирование оптических сигналов в различных спектральных диапазонах, включая создание детекторов „слепых“ к видимому диапазону [5-7]; селективное детектирование химических компонент и др. [8]. Преимущества, связанные с использованием органических материалов, основаны на широких возможностях современного органического синтеза, а также на развитии многочисленных низкотемпературных технологий формирования наноструктур на гибких полимерных подложках, включая метод печати [9]. В связи с этим особую важность приобретают и методы изучения таких структур.

В настоящей работе обсуждается метод широкополосной Фурье-спектроскопии, который может применяться не только для традиционного изучения спектральных свойств (пропускание, отражение, люминесценция и др.) органических структур, но и для изучения спектров их фотоэлектрического отклика, быстродействия и обнаружительной способности.

Рассматриваемый метод Фурье-спектроскопии основан на использовании гармонического закона модуляции оптической задержки, который был впервые предложен в Институте кристаллографии им. А.В. Шубникова РАН [10]. Особенностью этого способа модуляции оптической задержки является то, что он позволяет создавать достаточно простые Фурье-спектрометры, которые охватывают не только традиционный для Фурье-спектроскопии инфракрасный диапазон, но также видимую и УФ области спектра. В случае применения оптики из синтетического кварца и алюминиевых зеркал спектрометр способен охватывать диапазон длин волн от 4000 до $200 \mathrm{~nm}$, обеспечивая одновременно достаточно высокое спектральное разрешение $5-50 \mathrm{~cm}^{-1}$ во всем диапазоне. Легко реализуемое синхронное накопление интерферограмм позволяет получать высокое отношение сигнал/шум, что особенно важно для изучения систем на основе органических материалов, где сигнал может быть на много порядков ниже, чем в традиционных неорганических полупроводниках. В случае использования традиционных для видимого и УФ диапазонов дисперсионных спектрометров получение сравнимого отношения сигнал/шум требует применения методов фазочувствительного (синхронного) детектирования на каждой фиксированной длине волны и занимает существенно больше времени для регистрации всего спектра. Кроме того, при использовании дисперсионных спектрометров модуляция светового потока возможна лишь на относительно низкой частоте, ограниченной механическим модулятором, что не позволяет изучать быстродействие фотоэлектрических систем. В Фурье-спектроскопии модуляция светового потока на выходе интерферометра является естественным свойством прибора. При этом в нашем конкретном приборе частотным диапазоном этой модуляции можно легко управлять в широких пределах. Наконец, естественное присутствие в Фурье-спектроскопии 
средств спектрального анализа сигналов позволяет легко использовать метод для измерения таких важнейших свойств фотодетекторов, как удельная обнаружительная способность (specific detectivity) и эквивалентная мощность шума (noise equivalent power - NEP).

Работа организована следующим образом. Сначала мы приводим схему нашего Фурье-спектрометра и подробно рассматриваем особенности модуляции интенсивности светового пучка на выходе интерферометра Майкельсона, регистрацию и обработку интерферограмм для получения спектральных зависимостей фотоэлектрического отклика. Во второй части рассматривается конкретный пример изучения фотоэлектрического отклика в органической гетероструктуре, выполненного с использованием Фурье-спектрометра. Мы также сравниваем зависимости спектральной чувствительности, полученные методом Фурье, с зависимостями, измеренными классическим способом, используя дифракционный монохроматор. Последний раздел посвящен физической модели фотоэлектрического эффекта в органической гетероструктуре на основе фталоцианина цинка $(\mathrm{ZnPc})$ и фуллерена $\mathrm{C}_{70}$, построенной на основе полученных данных.

\section{Экспериментальная установка}

\section{Схема Фурье-спектрометра}

Экспериментальная установка включает четыре основных блока. Это источник света с коллиматором I, интерферометр Майкельсона II, система управления подвижным зеркалом и регистрации фотоэлектрических сигналов III, программное обеспечение для коммуникации с системой управления и регистрации, а также для обработки интерферограмм и вычисления Фурьеспектров (рис. 1).

Базовый блок источника света включает галогенную лампу 1 мощностью $50 \mathrm{~W}$ и коллимирующую кварцевую линзу 2 диаметром $50 \mathrm{~mm}$ и фокусным расстоянием $100 \mathrm{~mm}$. Галогенная лампа характеризуется гладким спектром излучения и хорошо подходит для исследований в спектральном диапазоне 350-2500 nm. Однако на длинах волн короче $350 \mathrm{~nm}$ количество света от галогенной лампы становится недостаточным для проведения измерений в широком динамическом диапазоне. Поэтому для проведения измерений в диапазоне 200-350 nm требуется использование иного источника света, например дейтериевой лампы (в нашем случае это лампа Hamamatsu L2D2 мощностью $30 \mathrm{~W}$ ).

После коллиматора близкий к параллельному пучок света попадает на вход интерферометра Майкельсона II, где он диафрагмируется (диаметр отверстия может варьировать в диапазоне от 30 до $2 \mathrm{~mm}$ ) и далее расщепляется делителем 4 на два пучка, которые соответственно отражаются от подвижного 5 и неподвижного 7 зеркал и интерферируют на выходе интерферометра. Делитель 4 выполнен в виде полупрозрачного алюминиевого зеркала, изготовленного вакуумным напылением алюминия

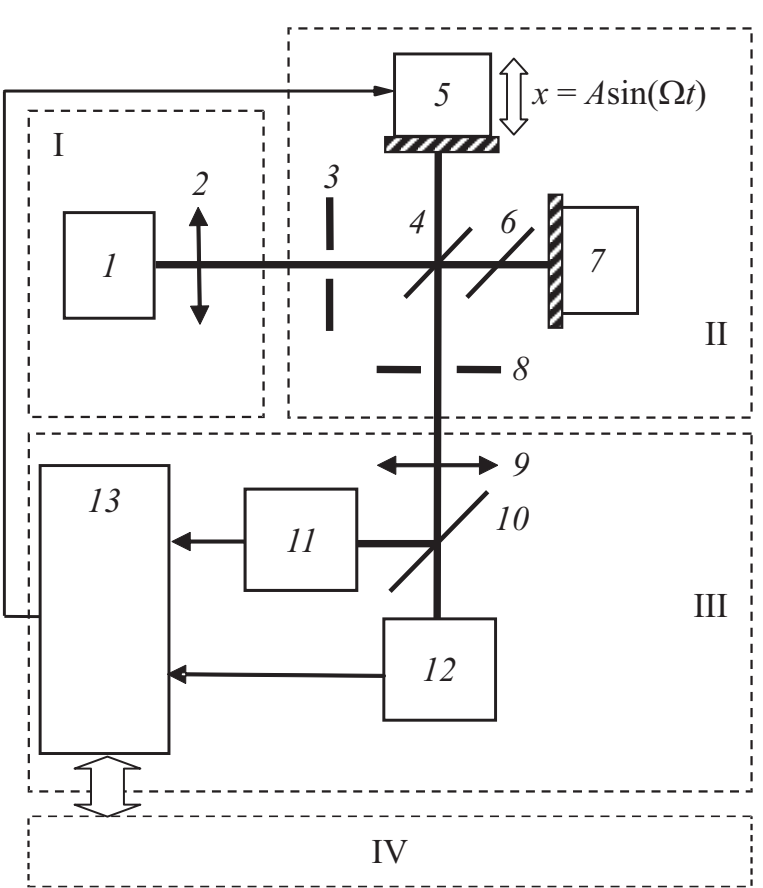

Рис. 1. Схема установки. I - блок источника света: 1 - галогенная или дейтериевая лампа, $2-$ кварцевый коллиматор; II - интерферометр Майкельсона: 3 - входная диафрагма, 4 - полупрозрачное алюминиевое зеркало на кварцевой подложке, 5 - подвижное алюминиевое зеркало, 6 - кварцевый компенсатор оптической дисперсии, 7 - неподвижное зеркало с микрометрической подачей, $8-$ выходная диафрагма; III - блок регистрации и управления: 9 - кварцевая линза, 10 - полупрозрачное алюминиевое зеркало на кварцевой подложке, 11 - опорный кремниевый фотодиод, 12 - исследуемый образец-фотоприемник, 13 - мультимедийная карта звукового интерфейса, включающая 24-разрядные двухканальные аналого-цифровые и цифро-аналоговые преобразователи; IV - программное обеспечение для персонального компьютеpa на базе операционной системы Microsoft Windows.

на кварцевую пластину. Наличие кварцевой пластины вносит спектрально зависимую оптическую задержку в плече интерферометра с подвижным зеркалом, что обусловлено наличием спектральной дисперсии показателя преломления кварцевой пластины. Чтобы скомпенсировать эту дисперсию в канал интерферометра с неподвижным зеркалом, вводится кварцевая пластина 6 , аналогичная той, на которой изготовлено полупрозрачное алюминиевое зеркало.

С выхода интерферометра (диафрагма 8) пучок попадает в блок регистрации и управления (III), где он фокусируется (кварцевая линза 9) и расщепляется (полупрозрачное зеркало 10) для регистрации интерферограмм фотоприемниками опорного 11 и основного 12 каналов. В качестве фотоприемника опорного канала используется кремниевый фотодиод с расширенной областью спектральной чувствительности $(200-1100 \mathrm{~nm})$. Фотоприемник основного канала - это исследуемый образец. 
Наличие опорного канала является принципиальным. Во-первых, интерферограмма, регистрируемая в этом канале, используется для автокалибровки прибора по шкале оптических частот (длин волн) и коррекции дрейфа интенсивности источника света. Во-вторых, наличие „опорной“ интерферограммы позволяет осуществлять синхронное накопление интерферограмм как в опорном, так и в основном канале и, таким образом, улучшать отношение сигнал/шум. Наконец, благодаря наличию опорного детектора спектральная чувствительность образца-фотоприемника может быть легко выражена в единицах чувствительности опорного фотодиода, а также в абсолютных единицах $(\mathrm{A} / \mathrm{W})$ при условии, что спектральная чувствительность фотоприемника опорного канала известна. Мы использовали фотодиод ФДУК-2УП производства АО „Техноэксан“ (Санкт-Петербург), спектральная чувствительность которого была предоставлена производителем.

Электрические сигналы от опорного и основного фотоприемников поступают на входы мультимедийной карты звукового интерфейса 13, которая используется для оцифровки сигналов с фотоприемников, а также для управления подвижным зеркалом. Взаимодействие между картой и персональным компьютером осуществляется с помощью специального программного обеспечения, созданного одним из авторов настоящей работы (С.П. Палто). В данной работе мы используем карту Creative E-MU 0204, которая содержит два входных канала с малошумящими усилителями сигнала (уровень шума $\sim 10 \mathrm{nV} / \mathrm{Hz}^{1 / 2}$ ) с 24-разрядным двухканальным аналого-цифровым преобразователем, а также двухканальным цифро-аналоговым преобразователем и соответствующим двухканальным усилителем с низким коэффициентом гармоник, который используется для управления подвижным зеркалом.

\section{Особенности модуляции интенсивности пучка в условиях интерференции на выходе интерферометра и восстановление оптического спектра}

На выходе интерферометра световой пучок является суперпозицией двух интерферирующих пучков. Если в спектре источника света выделить вклад на оптической частоте $\omega$, то электрическое поле данной спектральной компоненты на выходе интерферометра можно представить в виде

$$
E_{\omega}=E_{1} e^{-i \omega t-i k(x+\delta x)}+E_{2} e^{-i \omega t-i k x},
$$

где $E_{1}, E_{2}-$ амплитуды полей для компонент на частоте $\omega$ на выходе (диафрагма 8 на рис. 1) в двух интерферирующих пучках, прошедших через плечи интерферометра соответственно с подвижным и неподвижным зеркалами, $k$ - волновой вектор соответствующих компонент $(k=2 \pi / \lambda \equiv \omega / c$, где $\lambda$ - длина волны, $c-$ скорость света), $\delta x$ - смещение подвижного зеркала по отношению к началу координат $(x=0)$, соответствующему положению подвижного зеркала, при котором имеет место равенство плеч интерферометра.

Соответственно вклад в общую интенсивность от компоненты на частоте $\omega$ :

$$
\begin{aligned}
I_{\omega}=E_{\omega} E_{\omega}^{*} & =E_{1}^{2}+E_{2}^{2}+E_{1} E_{2} e^{-i k \delta x}+E_{1} E_{2} e^{i k \delta x} \\
& \equiv E_{1}^{2}+E_{2}^{2}+2 E_{1} E_{2} \cos (k \delta x),
\end{aligned}
$$

где символ „*“ означает комплексное сопряжение.

Таким образом, наряду с постоянной составляющей, описываемой первыми двумя членами выражения (2), интенсивность содержит переменную составляющую, определяемую изменением оптической задержки во времени $t(\delta x \equiv \delta x(t))$. В случае, когда $\delta x(t)$ изменяется во времени по линейному закону $(\delta x(t)=v t$, где $v-$ постоянная скорость движения зеркала), переменная составляющая интенсивности имеет вид

$$
\delta I_{\omega}=2 E_{1} E_{2} \cos (k v t) \propto I_{0, \omega} \cos (k v t),
$$

где $I_{0, \omega}-$ интенсивность спектральной компоненты на частоте $\omega$ в спектре излучения источника света. Таким образом, вклад каждой спектральной компоненты света оказывается промодулированным на выходе интерферометра на частоте $k v \equiv \omega v / c$. Для изменения во времени суммарной интенсивности, обусловленной всеми спектральными компонентами, в силу принципа суперпозиции имеет место соотношение

$$
\begin{aligned}
\delta I(t) & \propto \int_{\omega} I_{0, \omega} \cos \left(\frac{\omega}{c} v t\right) d \omega \\
& \equiv \frac{c}{v} \int_{\nu \omega / c} I_{0, \omega} \cos \left(\frac{\omega v}{c} t\right) d(\omega v / c) .
\end{aligned}
$$

Из выражения (4) легко видеть, что преобразование Фурье от $\delta I(t)$ дает спектр, подобный спектру излучения, где каждой компоненте на частоте модуляции интенсивности $(\omega \nu / c)$ соответствует спектральная компонента в спектре излучения на частоте $\omega$.

Сказанное выше хорошо известно и лежит в основе принципов работы классических Фурье-спектрометров [11]. В нашем случае ситуация иная. Оптическая задержка $\delta x(t)$ изменяется не по линейному, а по гармоническому закону:

$$
\delta x(t)=A \sin (\Omega t) .
$$

Соответственно скорость изменения оптической задержки

$$
v(t)=A \Omega \cos (\Omega t)
$$

более не является величиной постоянной, и применение преобразования Фурье непосредственно к зависимости интенсивности от времени $\delta I(t)$ для получения оптического спектра неприемлемо. Тем не менее эта 
проблема решается. Набор цифровых данных (исходная интерферограмма), взятых через равные интервалы времени, может быть преобразован в набор данных с переменным интервалом изменения $t$, который обеспечивает постоянное приращение задержки $\delta x$ так, что новая ,линеаризованная“ интерферограмма $\delta I(x)$ будет пригодна для получения оптического спектра, используя преобразование Фурье. Детальное рассмотрение процедуры преобразования данных интерферограммы не входит в задачи этой публикации. Отметим лишь, что в условиях, когда спектр сигнала ограничен, для получения ,линеаризованной“ интерферограммы исходные данные могут быть всегда корректно интерполированы со сколь угодно малым временным шагом $\delta t$ без потери исходной и внесения ложной информации о спектральных составляющих оптического спектра в силу хорошо известной теоремы Найквиста-ШеннонаКотельникова. Таким образом, интерферограмма $\delta I(x)$ может быть получена из $\delta I(t)$ применением процедуры интерполяции во времени с целью получения такого шага дискретизации $\delta t$, который обеспечивает достаточную точность представления данных с постоянным шагом $\delta x$, используя функцию арксинус (asin) [10].

Несмотря на необходимость математического преобразования исходных данных, гармонический закон изменения оптической задержки имеет множество преимуществ. Синусоидальный закон движения достаточно просто реализуется на практике, если, например, в качестве носителя зеркала использовать электродинамическую головку. При этом благодаря тому, что современные усилители звукового диапазона характеризуются очень низким коэффициентом гармонических искажений, можно обеспечить очень точное перемещение зеркала по синусоидальному закону. Последнее свойство позволяет отказаться от дополнительного лазерного канала в спектрометре и соответствующих сложных систем управления подвижным зеркалом со стабилизацией его скорости. Для калибровки прибора по частоте (длинам волн) достаточно использовать либо известную особенность в спектре излучения источника на известной длине волны, либо оптический фильтр с известным спектром.

При синусоидальном законе перемещения зеркала спектральное разрешение спектрометра легко варьируется изменением амплитуды колебаний $A$. В нашем конкретном примере значение $A$ может достигать $2 \mathrm{~mm}$, что соответствует разрешению $1 / A=5 \mathrm{~cm}^{-1}$ (на шкале длин волн $\delta \lambda \cong 0.13 \mathrm{~nm}$ при $\lambda=500 \mathrm{~nm})$. При частоте колебаний зеркала $\Omega=10 \mathrm{~Hz}$ регистрируется 20 спектров в секунду.

Варьируя амплитуду и частоту зеркала, можно также легко менять максимальную скорость модуляции интенсивности на определенной длине волны, что важно для изучения быстродействия (частотных характеристик) фотоэлектрического эффекта. Так, для максимальной частоты модуляции интенсивности в пучке на выходе интерферометра, соответствующей заданной длине волны $\lambda$, легко получить:

$$
f=A \Omega / \lambda .
$$

Например, при $A=1 \mathrm{~mm}$ и $\Omega=10 \mathrm{~Hz}$ максимальная частота изменения интенсивности спектральной составляющей на длине волны $500 \mathrm{~nm}$ равна $20 \mathrm{kHz}$. Если речь идет об измерении спектра в диапазоне длин волн от $4 \mu \mathrm{m}$ до $200 \mathrm{~nm}$, то это соответствует частотам модуляции от 2.5 до $50 \mathrm{kHz}$.

В нашем приборе частота колебаний зеркала $F=\Omega / 2 \pi$ может варьировать от 5 до $40 \mathrm{~Hz}$, а амплитуда $A$ от 50 до $1000 \mu \mathrm{m}$, что позволяет с хорошим спектральным разрешением в оптике перекрывать низкочастотный диапазон модуляции светового потока от единиц до сотен килогерц. В действительности, однако, в нашем конкретном случае мы ограничены максимальной частотой дискретизации аналого-цифрового преобразователя, которая составляет $192 \mathrm{kHz}$, и соответствующей этой частоте полосой пропускания до $96 \mathrm{kHz}$. Важно также, что при относительно высокочастотной модуляции светового потока схема включения измеряемого фотодетектора должна учитывать электрическую емкость образца так, чтобы не ограничивать время фотоэлектрического отклика параметрами внешней электрической цепи. Например, если образец-фотодетектор нагрузить активным сопротивлением $R=1 \mathrm{kOhm}$, которое существенно превышает его внутреннее сопротивление, то это нагрузочное сопротивление будет служить как преобразователь фототока в напряжение. При типичной емкости образцов $C \sim 4 \mathrm{nF}$ электрическая постоянная времени $R C$ при таком включении составляет $\sim 4 \mu \mathrm{s}$, что достаточно лишь для реализации верхней частотной границы $1 /(2 \pi R C) \sim 50 \mathrm{kHz}$.

\section{Эксперимент}

\section{Приготовление образца. Оптические и диэлектрические свойства}

Активный слой образца состоит из смесевой композиции фталоцианина цинка $\mathrm{ZnPc}$ (производства ГНЦ НИОПИК) с фуллереном $\mathrm{C}_{70}$ (производства Fullerene Centre). Смесь обоих материалов нанесена одновременно в вакууме $10^{-5}$ Torr на стеклянную подложку с прозрачным ITO (indium tin oxide)-электродом. При температуре тигля около $400^{\circ} \mathrm{C}$ напыляемое количество материала $\left(4 \mathrm{mg}\right.$ для $\mathrm{ZnPc}$ и $2 \mathrm{mg}$ для $\left.\mathrm{C}_{70}\right)$ использовалось полностью. Сверху на активный слой нанесен слой алюминия в виде полоски шириной $3.3 \mathrm{~mm}$, перпендикулярной полоске ITO-электрода аналогичной ширины. Таким образом, на подложке сформирована структура

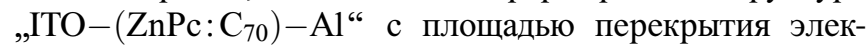
тродов $3.3 \times 3.3 \mathrm{~mm}$. Зависимость электрической емкости от частоты показана на рис. $2, a$. На участках подложки, свободной от электродов, измерялась оптическая 

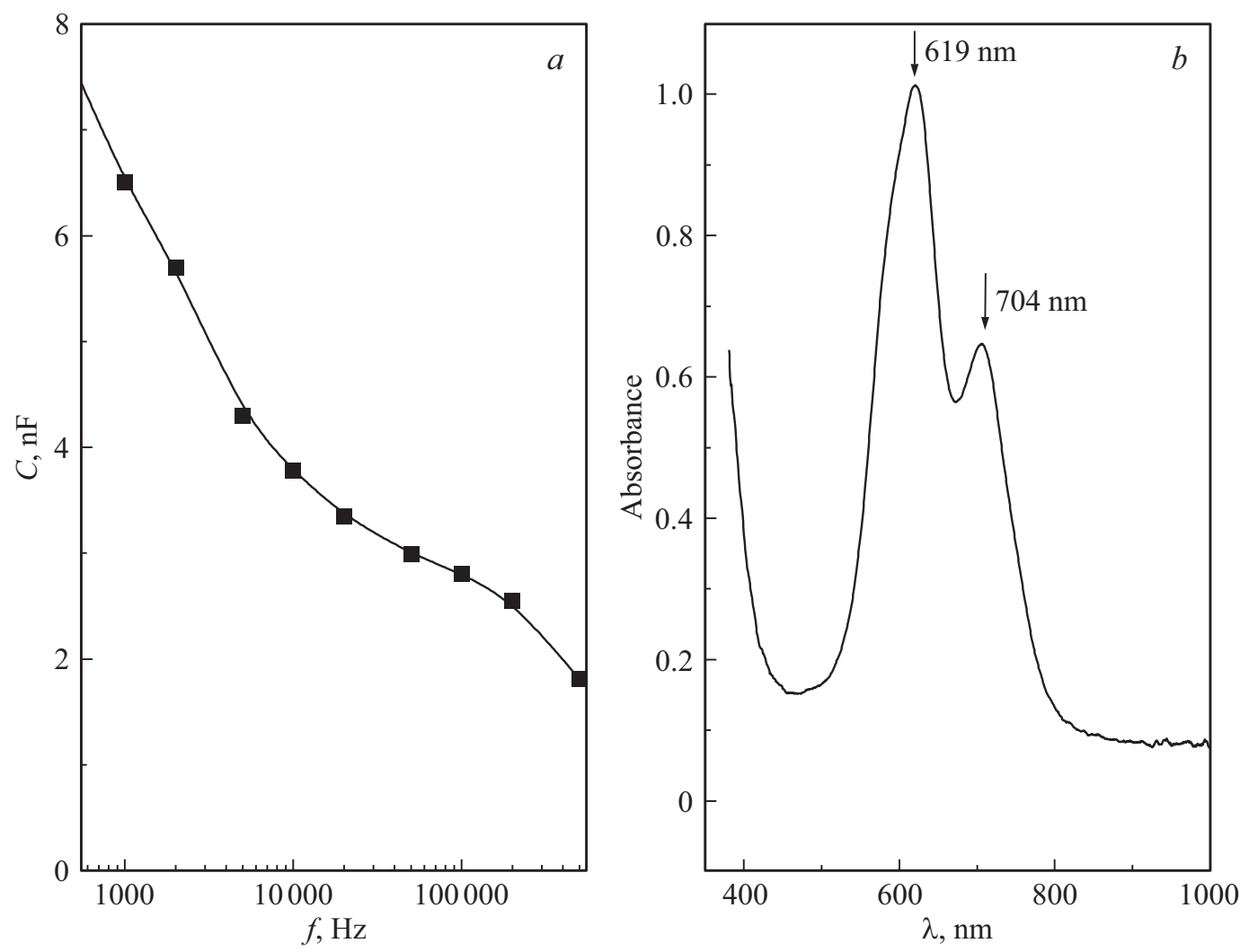

Pис. 2. (a) Частотная зависимость электрической емкости образца органической гетероструктуры; $(b)$ спектр поглощения слоя $\mathrm{ZnPc}: \mathrm{C}_{70}$.

плотность активного слоя (рис. 2, $b$ ). Следует отметить, что в настоящей работе нет каких-либо дополнительных слоев (например, PEDOT:PSS - poly(3,4-ethylenedioxythiophene)-poly(styrenesulfonate) [3]) или других проводящих покрытий. Толщина слоя $\mathrm{ZnPc}: \mathrm{C}_{70}$, измеренная с помощью интерферометра Линника, составляет $110 \pm 10 \mathrm{~nm}$.

Зависимость электрической емкости демонстрирует сильную частотную дисперсию как в области низких частот $(100-10000 \mathrm{~Hz})$, так и в области высоких частот (более $100 \mathrm{kHz}$ ). Это указывает на существование, как минимум, двух релаксационных процессов. Мы предполагаем, что низкочастотный релаксационный процесс обусловлен проводимостью, связанной с распределенным объемным зарядом по всей толщине органического слоя. Этот заряд может быть как собственным (возникающим благодаря полупроводниковым свойствам ZnPc и $\mathrm{C}_{70}$ ), так и инжектируемым из электродов. Релаксационный процесс на частотах выше $100 \mathrm{kHz}$ может быть связан с локальными случайно распределенными наноразмерными гетеропереходами $\mathrm{ZnPc}-\mathrm{C}_{70}$. Малые характерные размеры этих гетеропереходов по сравнению с толщиной всего активного слоя и обусловливают более высокочастотный характер этого релаксационного процесса.

Спектры поглощения ярко иллюстрируют полосы, характерные для электронных переходов в ZnPc (619 и $704 \mathrm{~nm}$, а также поглощение в ближнем УФ диапазоне). Однако поглощение $\mathrm{C}_{70}$, которое имеет место в диапазоне длин волн 380-500 nm, оказывается скрытым фоном сильного поглощения фталоцианина.

\section{Интерферограммы и оптические спектры фотоэлектрического отклика}

Кривая 1 на рис. 3, $a$ иллюстрирует интерферограмму света, зарегистрированную органическим детектором (образцом гетероструктуры $\mathrm{ZnPc}: \mathrm{C}_{70}$ ). Для сравнения на этом же рисунке показана интерферограмма, зарегистрированная кремниевым фотоприемником (кривая 2). Обе интерферограммы регистрировались на интервале движения зеркала от -160 до $+160 \mu \mathrm{m}$ $(x=0$ соответствует положению зеркала, при котором длины плеч интерферометра Майкельсона одинаковы). Такое перемещение зеркала обеспечивает спектральное разрешение $\sim 50 \mathrm{~cm}^{-1}$. Если сравнивать обе интерферограммы вблизи $x=0$, то обращают на себя внимание два принципиальных момента. Во-первых, максимальная амплитуда модуляции сигнала от образца гетероструктуры более чем на порядок меньше, чем от кремниевого фотодиода. Это свидетельствует о меньшей чувствительности гетероструктуры по сравнению с кремниевым фотоприемником. Во-вторых, энергия в интерферограмме от образца гетероструктуры менее локализована в окрестности $x=0$, что свидетельствует 

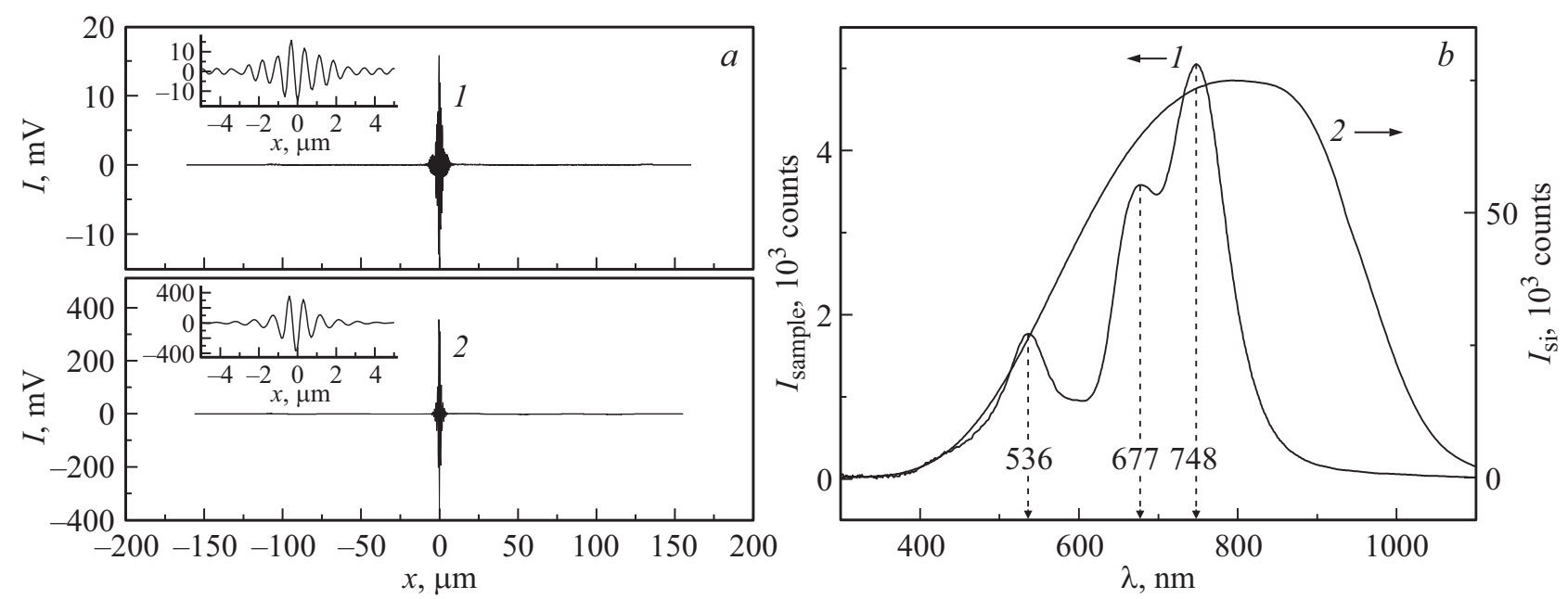

Рис. 3. (a) Экспериментальные интерферограммы, зарегистрированные образцом гетероструктуры 1 и кремниевым фотодиодом 2 ; (b) Оптические спектры фотоэлектрического отклика образца гетероструктуры (1, шкала слева) и кремниевого фотодиода $(2$, шкала справа).

о более узкой спектральной области чувствительности образца $\mathrm{ZnPc}-\mathrm{C}_{70}$ по сравнению с кремнием.

Bce сказанное детально иллюстрируется оптическими Фурье-спектрами фотоэлектрического отклика (рис. $3, b)$, которые получены из этих интерферограмм преобразованием Фурье. Как видно из этих спектров, спектральная область чувствительности органического детектора более узкая, чем у кремниевого фотодиода, а отклик в максимуме на длине волны $\lambda=748 \mathrm{~nm}$ примерно в 15 раз ниже, чем у кремниевого фотодиода. Весьма интересным является тот факт, что длины волн, соответствующие максимумам фотоэлектрического отклика $(748,677 \mathrm{~nm})$, не совпадают с длинами волн максимумов поглощения (704, $619 \mathrm{~nm}$, рис. 2, b), а находятся на длинноволновых краях этих полос поглощения. Кроме того, в спектре фотоэлектрического отклика ярко проявляются и полосы от $\mathrm{C}_{70}$ с максимумами на длинах волн 536 и $420 \mathrm{~nm}$, в то время как в спектрах поглощения полосы от $\mathrm{C}_{70}$ в области 500 и $400 \mathrm{~nm}$ скрыты сильным фоном поглощения молекул фталоцианина.

Спектры фотоэлектрического отклика суть произведение спектров спектральной плотности потока световой энергии на спектральную чувствительность фотодетектора. Так как интенсивность света от галогенной лампы сильно падает с уменьшением длины волны, то отклик сильно падает в области коротких длин волн. Однако поскольку Фурье-спектроскопия позволяет регистрировать спектры фотоэлектрического отклика в очень широком динамическом диапазоне (около 5 порядков величины благодаря синхронному накоплению интерферограмм), то даже в коротковолновом диапазоне спектр чувствительности гетероструктуры может быть легко выражен в относительных единицах чувствительности кремниевого детектора и даже в абсолютных единицах $(\mathrm{A} / \mathrm{W})$, так как ампер-ваттная характеристика кремниевого фотодиода известна.

\section{Ампер-ваттные спектральные характеристики и быстродействие}

На рис. 4 показаны спектры чувствительности органической гетероструктуры в абсолютных единицах $(\mathrm{A} / \mathrm{W})$, измеренные классическим способом с использованием дифракционного монохроматора (кривая 1) и с помощью Фурье-спектрометра (кривая 2). Различия между двумя спектрами сводятся лишь к разнице в амплитудных значениях отклика, что связано с ограниченным быстродействием нашего образца и будет обсуждаться ниже. Как видно, область наиболее высокой спектральной чувствительности детектора охватывает два диапазона — от 400 до $550 \mathrm{~nm} \mathrm{и} \mathrm{от} 650$ до $800 \mathrm{~nm}$. Следует

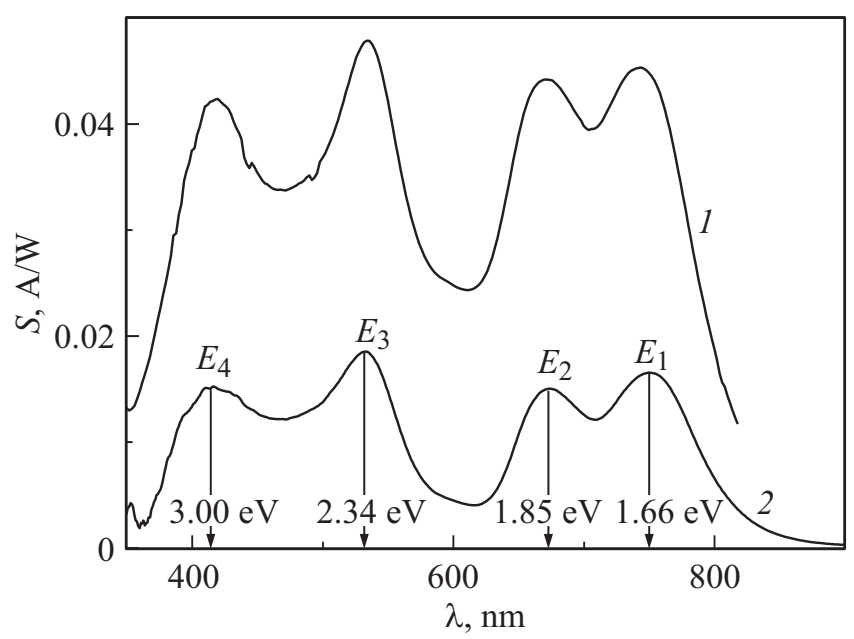

Рис. 4. Ампер-ваттные характеристики образца гетероструктуры, измеренные с использованием монохроматора МДР-23 при частоте модуляции светового потока $300 \mathrm{~Hz} 1$ и методом Фурье-спектроскопии 2 при частотной полосе модуляции светового потока в области частот $10-20 \mathrm{kHz}$. 

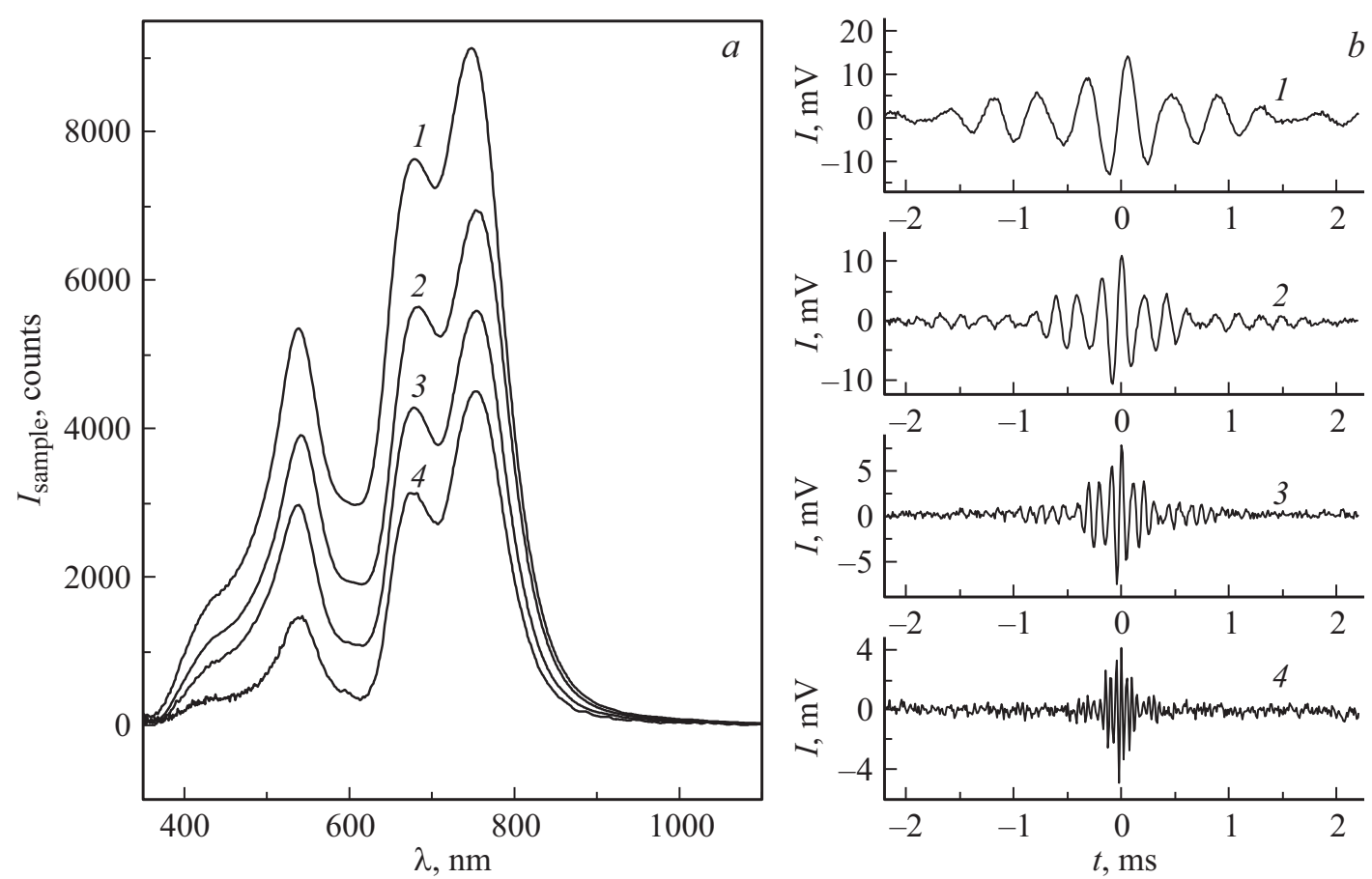

Pис. 5. Спектры Фурье фотоэлектрического отклика $(a)$ и соответствующие интерферограммы на временной шкале $(b)$, полученные при различных режимах управления подвижным зеркалом: варьируется параметр $A \Omega$ в соотношении (7), что соответствует различным частотным диапазонам регистрируемой модуляции интенсивности светового потока: кривые $1-2.5-5$, $2-5-10,3-10-20,4-20-40 \mathrm{kHz}$.

отметить, что в УФ диапазане на длинах волн короче $380 \mathrm{~nm}$ чувствительность детектора резко падает изза поглощения света стеклянной подложкой. Амперваттные характеристики показывают наличие четырех пиков чувствительности органического детектора. При этом в коротковолновом диапазоне чувствительность органического приемника лишь на порядок ниже, чем чувствительность кремниевого фотодиода, оптимизированного для УФ диапазона.

Показанные на рис. 3, $b$ и рис. 4 (кривая 2) спектральные зависимости были получены в условиях, когда модуляция светового пучка охватывает примерно частотный диапазон от 10 до $20 \mathrm{kHz}$. То есть наш органический фотодетектор демонстрирует относительно неплохое быстродействие. Однако так как электрическая емкость образца имеет сильную частотную зависимость, то возникает актуальный вопрос о соответствующей частотной зависимости (в данном случае имеется в виду не оптический, а низкочастотный диапазон от $100 \mathrm{~Hz}$ до $40 \mathrm{kHz}$ ) фотоэлектрического отклика. Как уже отмечалось выше, такие измерения можно провести благодаря возможности управления скоростью движения зеркала (см. формулы (6) и (7)), изменяя его амплитуду колебаний $A$ и частоту $F=\Omega / 2 \pi$. Измеренные оптические спектры отклика для разных диапазонов частот модуляции светового потока показаны на рис. 5.

Как видно, частотная зависимость проявляется достаточно ярко, хотя и не так сильно, как в случае фильтра низких частот первого порядка, когда изменение частоты в 10 раз приводит к падению сигнала тоже в 10 раз. В нашем случае при увеличении частоты в 10 раз (от 2.5 до $25 \mathrm{kHz}$, на длине волны $750 \mathrm{~nm}$ ) амплитуда отклика падает примерно в два раза. Это свидетельствует о достаточно сложной эквивалентной электрической схеме органического детектора, которая не описывается простой $R C$ цепью, характеризующей фильтр низких частот первого порядка. Относительно слабое падение фотоэлектрического отклика с ростом частоты позволяет говорить о нашем органическом детекторе, как о достаточно быстродействующем приборе.

Вернемся к сравнению (рис. 4) ампер-ваттных характеристик, измеренных классическим способом и Фурьеметодом. Кривая 1 измерена, используя монохроматор с дифракционной решеткой, механическим прерывателем пучка на частоте $300 \mathrm{~Hz}$ и фазочувствительным детектированием сигнала, а кривая 2 получена методом Фурье при интерференционной модуляции светового пучка на частотах в области $10-20 \mathrm{kHz}$. Как видно, спектральное поведение кривых с хорошей точностью идентично, а различие в абсолютных значениях амперваттной чувствительности хорошо согласуется с обсуждавшейся выше частотной зависимостью фотоэлектрического отклика.

Как уже отмечалось, максимумы спектральной чувствительности (рис. 4) не совпадают с максимумами поглощения $\mathrm{ZnPc}$, что с учетом литературных данных позволяет нам предположить, что эти максимумы соответствуют средним энергиям экситонных состояний 
$E_{1}=1.66 \mathrm{eV}, E_{2}=1.85 \mathrm{eV}, E_{3}=2.34 \mathrm{eV}, E_{4}=3.00 \mathrm{eV}$ в нанокристаллах фуллерена и фталоцианина. Если исходить из данных, приведенных для $\mathrm{C}_{70}$ в работе [12-14], то состояния $E_{2}-E_{4}$ следует отнести к экситонным состояниям и состояниям с переносом заряда в $\mathrm{C}_{70}$, а энергия $E_{1}=1.66 \mathrm{eV}$ соответствует ширине запрещенной зоны $\mathrm{C}_{70}[14]$. С другой стороны, энергии переходов $E_{1}, E_{2}$ очень близки к энергиям возбуждения 1.70 и $1.93 \mathrm{eV}$ максимального фотоэлектрического эффекта в пленке $\mathrm{ZnPc}$, измеренного нами в системе ITO-ZnPc-Al. Таким образом, энергии переходов $E_{1,2}$ присущи как $\mathrm{C}_{70}$, так и $\mathrm{ZnPc}$.

\section{Обнаружительная способность}

Согласно определению спектральная удельная обнаружительная способность (detectivity) $D(\lambda)$ фотоприемника определяется отношением ампер-ваттной спектральной чувствительности $S(\lambda)$ к среднеквадратичному (эффективному) значению спектральной плотности токового шума $I_{N}$ (обычно в единицах $\mathrm{A} / \mathrm{Hz}^{1 / 2}$ ):

$$
D(\lambda)=\frac{S(\lambda) \sqrt{A_{D}}}{I_{N}} \equiv \frac{S(\lambda) \sqrt{\left(A_{D}\right) R_{L}}}{U_{N}},
$$

где $A_{D}-$ площадь фоточувствительной области детектора, $U_{N}$ - эффективное напряжение шума, измеренное в частотной полосе шириной $1 \mathrm{~Hz}$ на нагрузке фотоприемника $R_{L}$ (в нашем случае для всех фотоприемников используется нагрузка $R_{L}=2 \mathrm{kOhm}$ ).

Еще одна важная характеристика, связанная с обнаружительной способностью, - это эквивалентная мощность шума в полосе $1 \mathrm{~Hz}$ :

$$
P(\lambda)=\frac{\sqrt{A}}{D(\lambda)}=\frac{I_{N}}{S(\lambda)} \equiv \frac{U_{N}}{R_{L} S(\lambda)} .
$$

Методы, заложенные в Фурье-спектроскопии, позволяют легко измерять значение $U_{N}$. В нашем случае массив данных темнового напряжения шума, измеренный на временном интервале в одну секунду с частотой дискретизации $96 \mathrm{kHz}$, подвергался преобразованию Фурье. В результате мы получали частотный спектр шума, который содержит 48000 независимых спектральных компонент шума с разрешением $1 \mathrm{~Hz}$. Равномерное распределение шума по частотам позволяет провести статистическую обработку спектральных данных и с достаточно высокой точностью определить среднеквадратичные значения напряжения шума $U_{N}$ с достаточно высокой точностью $\sim 0.1 \mathrm{nV}$.

Так как вклад в шум вносит не только фотодетектор, но и измерительный усилитель с сопротивлением нагрузки, то шум от фотодетектора определяется как

$$
U_{N}=\sqrt{U^{2}-U_{0}^{2}-4 k T R_{L}},
$$

где $U_{0}$ - эффективное значение шума измерительного усилителя (когда фотодетектор не подключен к усилителю), $U$ - шум усилителя с подключенным фотодетектором и нагрузочным сопротивлением, $T-$ абсолютная температура, а $k$ - постоянная Больцмана. Из (10) с учетом измеренного шума усилителя получено значение $U_{N} \cong 0.6 \mathrm{nV}$, а с учетом $S=0.02 \mathrm{~A} / \mathrm{W}$ эквивалентная мощность шума $P=1.5 \cdot 10^{-11} \mathrm{~W}$. Следует отметить, что из-за значительного шума от сопротивления нагрузки (эффективное значение шума Джонсона $\sim 5.7 \mathrm{nV})$ относительная погрешность определения $U_{N}$ оказывается значительной $(\sim 20 \%)$. Используя данные на рис. $4(S=0.02 \mathrm{~A} / \mathrm{W}$ на длине волны $530 \mathrm{~nm}$, $U_{N}=0.6 \mathrm{nV}$ ) и применяя формулу (8) с учетом того, что фотоактивная площадь органической гетероструктуры составляет $0.11 \mathrm{~cm}^{2}$, в области частот $5-10 \mathrm{kHz}$ получаем $\left.D\right|_{\lambda=530 \mathrm{~nm}, f=10 \mathrm{kHz}}=2 \cdot 10^{10} \mathrm{~cm} \cdot(\mathrm{Hz})^{1 / 2} / \mathrm{W}$. Хотя аналогичные оценки для кремниевого фотодиода ФДУК-2УП, имеющего примерно в 3 раза большую фотоактивную площадь и более высокую ампер-ваттную чувствительность, приводят к существенно большей обнаружительной способности, полученное значение удельной обнаружительной способности для органической гетероструктуры достаточно высокое, чтобы использовать ее в качестве фотодетектора излучения видимого спектрального диапазона.

\section{Модель фотоэлектрического эффекта}

Полученные нами данные об энергиях возбуждения фотоэлектрического эффекта позволяют построить энергетическую диаграмму возбужденных состояний в объемной гетероструктуре $\mathrm{ZnPc}: \mathrm{C}_{70}$ между электродами ITO и алюминия. На рис. 6 показана соответствующая диаграмма в состоянии термодинамического равновесия, когда уровень Ферми $\left(E_{F}\right)$ один и тот же для элементов всей системы. Так как работы выхода электрона из ITO $(4.8 \mathrm{eV})$ и $\mathrm{Al}(4.2 \mathrm{eV})$ сильно различаются, то в состоянии термодинамического равновесия имеется избыточный положительный заряд вблизи $\mathrm{Al}$ и, соответственно, отрицательный заряд у ITO-электрода. Эти заряды создают встроенное электрическое поле [15], которое охватывает почти всю толщину слоя $\mathrm{ZnPc}: \mathrm{C}_{70}$ (исключение составляют лишь приэлектродные области, где знак поля должен быть противоположный, чтобы обеспечить нулевую разность потенциалов на всей структуре) и играет очень важную роль в транспорте фотоэлектрических зарядов. Отметим, что наличие встроенного поля не приводит к темновому электрическому току, так как дрейфовый ток, обусловленный полем, компенсируется диффузионным током, связанным с градиентом концентрации носителей заряда в объеме пленки. На диаграмме наличие встроенного поля отражается наклоном прямой, соединяющей точки потенциалов вакуума вблизи ITO- и $\mathrm{Al}$ электродов.

Из-за смесевого способа приготовления слоя $\mathrm{ZnPc}: \mathrm{C}_{70}$ в объеме пленки существует множество нанокристаллических областей, где молекулы ZnPc контакти- 


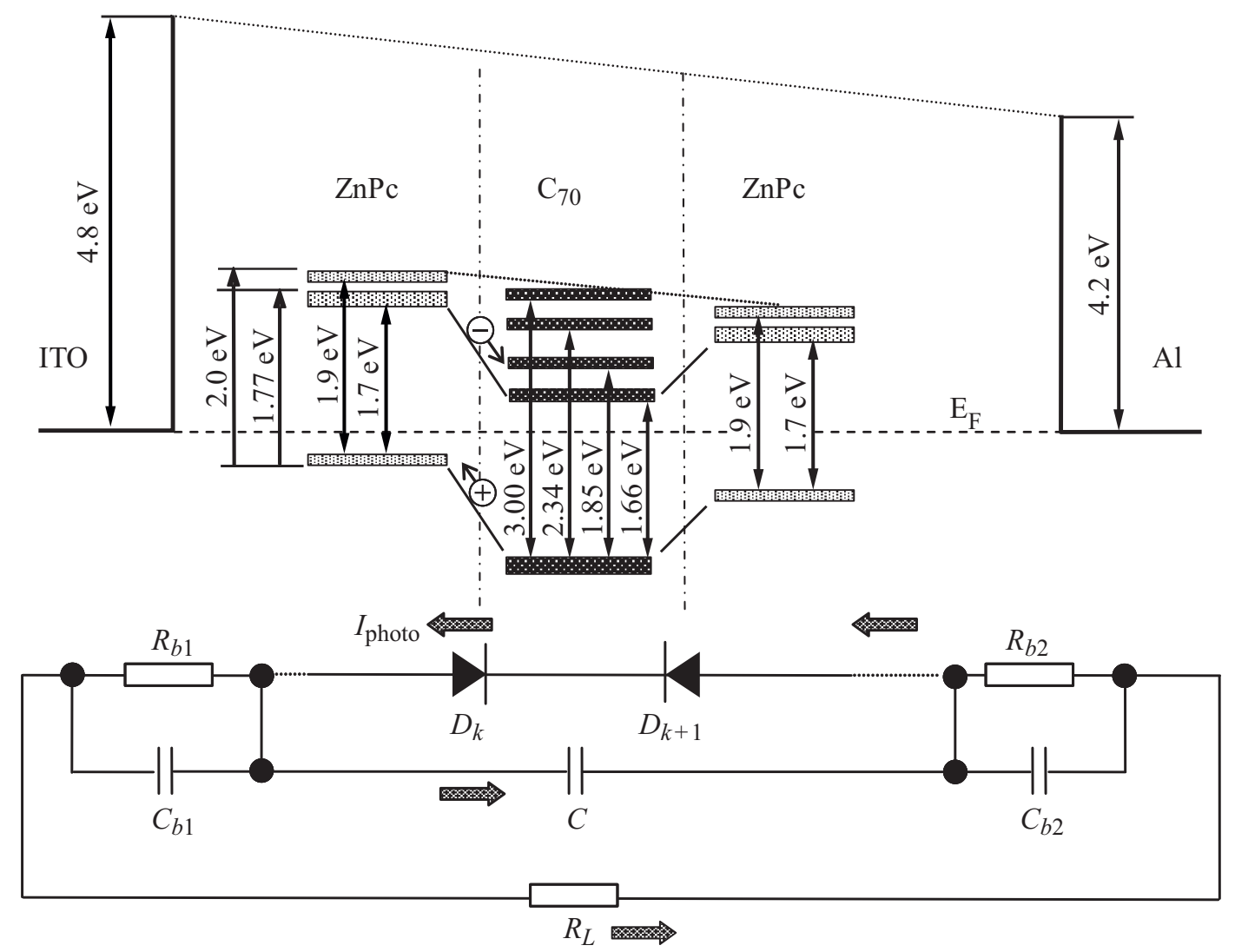

Рис. 6. Диаграмма энергетических уровней в системе ITO-ZnPc: $\mathrm{C}_{70}-\mathrm{Al}$ и эквивалентная электрическая схема фотоприемника органической гетероструктуры.

руют с молекулами $\mathrm{C}_{70}$, образуя гетеропереходы. Для наглядности на рис. 6 показаны два таких гетероперехода.

B ZnPc переходы 1.77 и $2.0 \mathrm{eV}$ соответствуют расщепленному электронному переходу [16], который проявляется в спектрах поглощения как хорошо известная Q-полоса. Ниже этих расщепленных уровней лежат зоны с энергиями переходов вблизи 1.7 и $1.9 \mathrm{eV}$, которые мы связываем с экситонными состояниями. Эти энергии были определены нами в независимом эксперименте по измерениям спектрального положения максимумов фотоэлектрической чувствительности в чистых пленках ZnPc. При этом мы предполагаем, что самая нижняя экситонная зона перекрывается с зоной проводимости $\mathrm{ZnPc}$ и, таким образом, относим значение энергии $1.70 \mathrm{eV}$ к ширине запрещенной зоны в $\mathrm{ZnPc}$.

Энергии четырех уровней $(1.66,1.85,2.34,3.00 \mathrm{eV})$ в $\mathrm{C}_{70}$ соответствуют максимумам фоточувствительности в исследованном нами образце (рис. 4). Согласно литературным данным, эти энергии принадлежат экситонным уровням и ширине запрещенной зоны $(1.66 \mathrm{eV})$.

Так как, согласно данным [17], поликристаллический фталоцианин цинка проявляет свойства полупроводника p-типа, то уровень Ферми в ZnPc оказывается ближе к валентной зоне. А поскольку фуллерены относятся к полупроводникам $n$-типа [12], то в области $\mathrm{C}_{70}$ уровень Ферми находится ближе к зоне проводимости. Таким образом, в результате выравнивания уровня Ферми в областях контакта $\mathrm{ZnPc}$ и $\mathrm{C}_{70}$ возникают $p-n$-переходы (диоды) с сильным встроенным локальным полем, которое на наш взгляд играет ключевую роль в диссоциации экситонных возбуждений на электронно-дырочные пары и разделении зарядов.

Встроенное электрическое поле, связанное с разностью работ выхода, смещает эти локальные гетеропереходы. При этом в половине гетеропереходов $\mathrm{ZnPc} \rightarrow \mathrm{C}_{70}$ (в данном случае стрелка указывает направление от ITO к Al) это смещение усиливает локальное поле гетероперехода, смещая соответствующий диод в обратном направлении. Вторая половина диодов $\left(\mathrm{C}_{70} \rightarrow \mathrm{ZnPc}\right)$ оказывается смещенной в прямом направлении. Если первый тип диодов $\left(D_{k}\right)$, смещенных в обратном направлении, склонен к генерации фотоэлектрических зарядов, то второй тип диодов $\left(D_{k+1}\right)$ склонен просто к большей проводимости.

В итоге мы предлагаем эквивалентную электрическую схему, показанную снизу на рис. 6. В этой схеме генераторами фотоэлектрического тока является примерно половина от всех локальных фотодиодов в общей объемной гетероструктуре. Эти диоды смещены встроенным полем, возникающим от разности работ выхода, в обратном направлении (один из этих фотодиодов обозначен на схеме как $D_{k}$ ). Вторая половина фотодиодов, таких 
как $D_{k+1}$, могла бы генерировать фототок обратного знака, но, будучи смещенными в прямом направлении, они просто выступают в роли проводников.

Наличие гетеропереходов и объемного свободного заряда приводит к достаточно сложной природе электрической емкости $C$. Эта емкость определяется не только геометрией образца, но и распределением и количеством

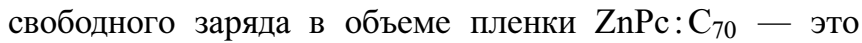
так называемая диффузионная емкость. Кроме объемной емкости в нашей системе также существуют барьерные (контактные) емкости $C_{b 1}$ и $C_{b 2}$. Эти емкости образуются из-за рассогласования положения зон проводимости и валентных зон в гетероструктуре $\mathrm{ZnPc}: \mathrm{C}_{70}$ по отношению к уровням Ферми в металлических электродах. Как уже отмечалось, выравнивание уровня Ферми сопровождается инжекцией зарядов и возникновением встроенного поля и соответствующих диодов (в данном случае диодов Шоттки, которые мы не показываем на схеме; из-за смесевого состава пленки у каждого из электродов возникает два типа диода Шоттки, например $\mathrm{C}_{70}-\mathrm{Al}$ и $\mathrm{ZnPc}-\mathrm{Al}$ ). Наличие диодов Шоттки с неизбежностью предполагает возникновение обедненных зарядом приэлектродных областей с пониженной проводимостью, определяемой сопротивлениями $R_{b 1}$ и $R_{b 2}$ в нашей эквивалентной схеме.

Предложенная эквивалентная схема позволяет качественно понять как частотную зависимость электрической емкости нашего образца (рис. 2), так и частотную зависимость фотоэлектрического отклика (рис. 5). Действительно, на низких частотах, когда импеданс приэлектродных слоев определяется лишь сопротивлениями $R_{b 1}$ и $R_{b 2}$, определяющей является объемная емкость $C$ (в нашем случае $\sim 7 \mathrm{nF}$ ), которая включает как геометрическую емкость органической гетероструктуры, так и диффузионную емкость, связанную с объемным зарядом в ней. С повышением частоты поля импеданс барьерных слоев начинает в большей степени определяться их барьерными емкостями, а диффузионная емкость постепенно „выключается“", так как объемный заряд не успевает следовать за переменным полем. В итоге на высоких частотах емкость определяется последовательно соединенными барьерными и объемной геометрической емкостями, что выражается в падении общей емкости от $7 \mathrm{nF}$ (на частоте $100 \mathrm{~Hz}$ ) до $2 \mathrm{nF}$ на частоте $100 \mathrm{kHz}$. Что касается фотоэлектрического отклика, то на постоянном токе все емкости $\left(C, C_{b 1}\right.$, $\left.C_{b 2}\right)$ „выключены“ и фотоэлектрическое напряжение на нагрузочном сопротивлении $R_{L}$ зависит от барьерных сопротивлений $R_{b 1}, R_{b 2}$, которые могут быть достаточно большими и сильно подавлять фотоэлектрический отклик на сопротивлении нагрузки. Однако на переменном токе с повышением частоты $f$ электрический импеданс барьерных слоев падает, т. е. по мере повышения частоты активные сопротивления $R_{b 1}, R_{b 2}$ перестают играть значимую роль, так как они оказываются существенно больше, чем емкостные сопротивления $1 /\left(2 \pi f \cdot C_{b 1, b 2}\right)$. Именно этот фактор вместе с падением емкости $C$ за счет „выключения“ диффузионной емкости способствуют наблюдаемому замедлению падения электрооптического отклика с ростом частоты.

\section{Заключение}

Таким образом, предложенный метод изучения фотоэлектрических эффектов обладает широким спектром возможностей, которые включают измерения спектральной чувствительности, быстродействия и обнаружительной способности фотодетекторов. Предложенный метод Фурье-спектроскопии, основанный на гармоническом законе модуляции оптической задержки в интерферометре Майкельсона, характеризуется широким спектральным и динамическим диапазонами, гибкостью управления низкочастотным спектром модуляции интенсивности светового пучка, которые трудно реализуемы на базе дисперсионных спектрометров, а также на основе Фурьеспектрометров с традиционным способом изменения оптической задержки.

Нами подробно изучены фотоэлектрические свойства конкретной органической гетероструктуры: определены энергии переходов, ответственных за максимальный фотоэлектрический отклик, предложена модель и эквивалентная электрическая схема органического фотодетектора ITO-ZnPc: $\mathrm{C}_{70}-\mathrm{Al}$, который продемонстрировал свойства, пригодные для практических приложений.

Работа выполнена в рамках проекта РНФ № 14-12-00553.

\section{Список литературы}

[1] Blinov L.M. Structure and Properties of Liquid Crystals. Springer, 2011.

[2] Chang Y.-L., Lu Z.-H. // J. Disp. Technol. 2013. V. 9. N 6. P. 459. doi 10.1109/JDT.2013.2248698

[3] Manna E., Xiao T., Shinar J., Shinar R. // Electronics 2015. V. 4. P. 688. doi 10.3390/electronics 4030688

[4] McGehee D.G., Topinka M.A. // Nature Mater. 2006. V. 5. N 9. P. 675. doi 10.1038/nmat 1723

[5] Yu G., Pakbaz K., Heeger A. // J. Appl. Phys. Lett. 1994. V. 64. P. 3422. doi 10.1063/1.111260

[6] Peumans P., Uchida S., Forrest S.R. // Nature 2003. V. 425. P. 158. doi 10.1038/nature01949

[7] Lin H.-W., Ku S.-Y., Su H.-C., Huang C.-W., Lin Y.-T., Wong K.-T., $W u$ C.-C. // Adv. Mat. 2005. V. 17. P. 2489. doi 10.1002/adma.200401622

[8] Hofmann O., Miller P., Sullivan P., Jones T.S., deMello J.C., Bradley D.D.C., deMello A.J. // Sens. Actuators B. 2005. V. 106. P. 878. doi 10.1016/j.snb.2004.10.005

[9] Aernouts T., Aleksandrov T., Girotto C., Genoe J., Poortmans J. // Appl. Phys. Lett. 2008. V. 92. P. 033306. doi 10.1063/1.2833185

[10] Палто С. П., Блинов Л.М., Яковлев С.В., Вологин В.И., Шилин Ю.Н. Патент РФ № 2239801, 2004.

[11] Вагин В., Гершун М., Жижин Г., Тарасов К. Светосильные спектральные приборы. М.: Наука, 1986. 
[12] Макарова Т.Л. // ФТП. 2001. Т. 35. № 3. С. 257.

[13] Kazaoui S., Minami N., Tanabe Y., Byrne H.J., Eilmes A., Petelenz P. // Phys. Rev. B. 1998. V. 58. N 12. P. 7689. doi 10.1103/PhysRevB.58.7689

[14] Zhou W., Xie S., Qian S., Zhou T., Zhao R., Wang G., Qian L., Li W. // J. Appl. Phys. 1996. V. 80. P. 459. doi $10.1063 / 1.362747$

[15] Лазарев В.В., Блинов Л.М., Юдин С.Г., Палто С.П. // ЖЭТФ. 2014. Т. 146. C. 854. 10.7868/S0044451014100204; Lazarev V.V., Blinov L.M., Yudin S.G., Palto S.P. // JETP. 2014. V. 119. P. 753.

[16] Morley J.O., Charlton M.H. // J. Phys. Chem. 1995. V. 99. P. 1928. doi 10.1021/j100007a023

[17] Pfuetzner S., Meiss J., Petrich A., Riede M., Leo K. // Appl. Phys. Lett. 2009. V. 94 P. 223307-3. doi 10.1063/1.3148664 